\title{
Collaborative Services for Customized Production in Networked Companies
}

\author{
Rosanna Fornasiero ${ }^{1}$, João Bastos ${ }^{2}$, Américo Azevedo², \\ Andrea Zangiacomi ${ }^{1}$, and Eva Coscia ${ }^{3}$ \\ ${ }^{1}$ ITIA-CNR -Institute of Industrial Technologies and Automation - National Council of Research, \\ Via Bassini 15, 20133 Milano, Italy \\ \{rosanna. fornasiero, andrea.zangiacomi\} eitia.cnr. it \\ ${ }^{2}$ INESC TEC (formely INESC Porto) \& Faculdade de Engenharia da Universidade do Porto, \\ Rua Doutor Roberto Frias 378, 4200-465 Porto, Portugal \\ \{jsbastos, ala\}@fe.up.pt \\ ${ }^{3}$ TXT e-Solutions SPA, Via Frigia 27, 20126 Milano, Italy \\ eva.coscia@txtgroup.com
}

\begin{abstract}
Increasingly, consumer demand of fashionable products is arising as significant challenge for company managers. In order to respond to this demand, companies are asked to supply small series of innovative and fashionable goods of high quality, affordable price and eco-compatibility in short periods of time and with high service levels. As a result of these rapidly evolving challenges, companies are forming collaborative networks in order to design, develop, produce and distribute such products and services in a collaboratively way. These highly integrated and dynamic supply networks depend intensively in new set of tools, methods and related services in which rely the collaborative networking operation. This paper presents a specific case study implementation of these collaborative supporting services, through the realization of a collaborative portal.
\end{abstract}

Keywords: Collaborative Networks, Collaborative Portal, Supply Networks.

\section{Introduction}

Due to social, technological, economical and financial changes, company managers need to re-invent the concept of enterprise. A new level of complexity emerged in the last decade, forcing managers to address the market and especially the individual customer with augmented care by putting more emphasis on the service levels they provide, by reducing response times and by tackling the specific needs of the diversity of customers. Added to this pressing necessity, the managers are realizing the fact that competition as well as collaboration schemes are transitioning from among companies to among supply networks, and management of both inter-organizational and intersupply chain processes and information is even more critical for rapid response and quality assurance of products and processes specially when producing in demand driven supply networks.

Along this vein, consumer needs and expectations of specific target groups - such as elderly, obese, disabled, or diabetic persons - are arising as challenging 
opportunities for European companies which are asked to supply small series of innovative and fashionable goods of high quality, affordable price and eco-compatible in short periods of time and with high service levels. In order to design, develop, produce and distribute such products and services in a collaboratively way, a new set of tools, methods and related components of collaborative networking are required.

The main objective of the present paper, framed within the EU (European Union) funded project CoReNet, is to present an innovative Collaborative Portal for the TCF SMEs (small and medium enterprises) companies in collaborative networks to support the network formation, the knowledge sharing and the expertise in manufacturing of small batches of products addressing the need of the special consumer target groups. The aim of this collaborative portal is to equip fashionable and healthy footwear \& garments network managers and stakeholders with the necessary guidance to address the combination of processes, functions, activities, relationships and pathways along which products, services and information move in and between TCF companies.

The remaining of this paper is organized as follows: first the existing related literature is shown as theoretical foundations, followed by an enumeration of the required services for collaborative networks. Finally, conclusions and final remarks are presented concerning the collaborative portal and the following steps regarding their development and improvement.

\section{Foundations and Research Topics}

Managerial Reasons. In recent years, it is emerging at industrial level, the awareness that full adoption of collaborative small series production methodologies and technologies are of decisive importance to European Manufacturing Industry, SMEs. In reality, companies urgently need to proactively respond to the high variability of the consumers demand and expectations, to reduce the risks of following too fast changing trends without appropriate basis, which cause serious limits in terms of both customer's satisfaction and enterprises competitiveness and sustainability This is especially true in consumer goods sectors, where customers' tastes change very quickly, and even more in the current economic situation of downturn, where the purchase capability of customers has sharply decreased and is this strongly focused on the high value products. In particular for fashionable sectors such as Textile \& Clothing Industry and Leather and Footwear Industry (TCFI) the number of seasonal collections has been increasing enlarging the offer in terms of models along the year, and the number of sold products is decreasing in major markets, leaving best opportunities only for value added products. Moreover, social phenomena like ageing, increase of obese people and major sensitivity versus disabled people and versus ecofriendly products, are also key challenges to be faced by the considered consumers sectors, with reference to healthcare and sustainability. In order to address the new type of target groups (elderly, obese, disabled, or diabetic persons) demand, it is necessary to develop new collaborative supply chain solutions based on cost effective, social compliant and eco-efficient design and production of customized products that fully satisfy the customers, considering their health issues as well as their desire for fashionable products. 
Recent research in the field addressed different forms of business networks and their structural forms of organization. The literature distinguishes for example by value chain orientation (horizontal, vertical, lateral), life span (long-term vs. short-term), and degree of virtualization or hierarchical structure (hierarchical vs. non-hierarchical networks) [1]. Nevertheless, the most common business networks are formed along the value chain and for enduring purposes [2]. In parallel, the current market trends demands for flexibility at the supply network level, the processes and the product designs in order to empower the companies to quickly adapt for new business requirements and sustainability challenges. This new demands are forcing business networks to have much shorter life-time existence and take advantage of new infrastructure technologies supported in distributed information systems and knowledge [3].

A new concept of demand-driven supply networks is emerging in literature as a collaborative approach in response to consumer's needs and expectations. In this new demand paradigm the value creation has shift from the traditional idea of value creation and has become co-creation, where customers actively co-create and recreate value with organisations [4]. Thus, all these changes are setting the stage for an expanded role for customers, changing the balance of power and forcing companies to provide new co-creation environments, where customers are no longer passive recipients of goods and services and instead customers are now active partners cocreating value with organisations [5]. In reality, many companies that embrace this paradigm transformed their operating systems from the traditional functional supply networks through a holistic approach that addresses demand and customer expectations in all of its dimensions. This implies different approaches to the market based not only on traditional sales channels (shops, retailers) but increasingly on an Internet mediated direct contact with consumers for product co-design and coinnovation, for product sales and consumers marketers support but also for after-sales services. Concurrently, consumers are increasingly valuing collaborative networks that endorse the sustainability challenges. These networks by seeing the world's present and future challenges seek to develop new products and processes that can be part of a sustainable solution. Namely, through a holistic view of the supply network it is possible to measure and optimize the overall impact of the "carbon footprint", to implement policies that seek recycling and waste prevention, product design for sustainability and the use of emerging clean technologies [3].

One of the major concerns in sustainable supply chain has been the supplier management. Several recent incidents related with sustainability have shown that the reason suppliers cause such environmental problems is because most suppliers are small and medium-sized enterprises (SMEs) which cannot sufficiently deal with the environmental management issues. These cases show how important supplier selection and support are. Even if such mistakes occurred in the upstream of a buying firm's supply chain, it is the buying firm's responsibility to the public. Such incidents cause damages to buying firms financially as well as damages on reputation [6].

From the production viewpoint, companies from different sectors in sustainable networks need to integrate their production systems in order to offer to the customer integrated solutions and innovative services and products.

Perspectives on Collaborative Business Networks . Growing competitiveness in the global market encourages manufacturing companies to form alliances among them for 
mutual benefit. Various forms of collaboration emerged as response to transformations in the business environment and the rapid developments in information and communication technologies (ICTs) [7]. Collaboration in the form of virtual organizations [8-9], virtual organization breeding environments (VBE), business community [10], business ecosystems [11], etc. are a powerful instrument to achieve strategic objectives such as short lead time, high quality, and cost competitiveness especially for SMEs which need to create critical mass to stay competitive. The multi-perspective approach on collaborative environment (technological, semantic, social and business perspective) emphasizes the importance of the business view that allows collaborative networks to be regarded as combinations of inter and intra-organizational business processes [12].

Forming Virtual Organizations (VO) can offer a new competitive environment and manufacturing agility [13-14). The VO is a temporary alliance of member companies which join to take advantage of market opportunities [15-16] as specific target market requirements can be. Within a $\mathrm{VO}$, each member company will provide its own core competencies in areas such as research and development, marketing, engineering and manufacturing. One of the major issues in the formation and operation of VO is the rapid integration of the business processes of collaborative companies.

During business networking, it is critical that strategic collaborative relationships between partner organizations are developed both upstream and downstream the dynamic business environment. It is expected that participating companies must continue to revamp their business model in response to industry competitions and product lifecycle. From the perspective of SMEs, the collaborative business is an alternative to traditional supply chains, where they can manage the business better and increase their added value [17-19]. In regional or sectorial Business Communities, trust can be built and communication streamlined, creating an environment suitable for the fast and efficient creation of Virtual Organizations (partnerships or collaboration projects) to respond to specific business opportunities [9].

ICT Requirements. In order to develop a collaborative platform to support the intended high levels services it is necessary to start from the main requirements within the different user domains (manufacturer, suppliers, service partners, customers, etc.) as well as the technical requirements related to the software modules and involved data flows (and repositories). Also the middleware components devoted to provide general services to be used from different applications (authentication, workflow management and business document engines; product data handling, etc...).

A suitable approach to build the necessary architecture to support collaborative portal for customized production in networked companies is the Service Oriented Architecture (SOA). The SOA is an emerging approach that addresses the requirements of loosely coupled, standards-based, and protocol independent distributed computing. Typically business operations running in an SOA comprise a number of invocations of these different components, often in an event-driven or asynchronous fashion that reflects the underlying business process needs [20].

Service Oriented Architecture (SOA) is a flexible set of design principles used during the phases of systems development and integration. A system based on a SOA will package functionalities as a suite of interoperable services that can be used within multiple separate systems from several business domains. SOA architectures are 
generally based on several elements: (i) Services (usually web-services): they represent atomic functionalities offered by the system. (ii) Vehicle messages: they represent the information exchanged by the different services. They are based on XML. (iii) Enterprise Service Bus (ESB): it is the channel used to integrate together all the services and exchange messages.

Nowadays SOA solutions are very popular, because they offer several advantages over traditional architectures: (i) Loose coupling: services are no more directly connected each other, but instead they exchange messages using a service bus. This allows services to be updated and substituted without heavy impact on the whole architecture. (ii) Flexibility: the backbone of a SOA architecture is represented by the ESB. Adding or removing services and functionalities becomes very easy since it is just a matter of configuring the bus, while the services are not touched by these operations. (iii) Easy maintenance: functionalities offered by SOA are fruited using web services. End users don't need to install anything to use the services, and the maintenance is centralized in the hand of the developers. This allows end users and providers to cut dramatically the costs related to update and maintenance of software.

These three advantages, together with the easy configuration of modern Enterprise Service Bus (ESB) and development of services, decreed the success of SOA architectures. Another relevant ICT aspect in building and providing an infrastructure for collaborative networks is related with the use of open-source software solutions. As Frank Hecker point out, in the traditional software business models, softwarehouses provides all (or almost all) of the value to customers, and they realize their revenues and profits in return through traditional software license fees. On the other hand, in an open-source business model, much of the value provided to customers will not be provided solely by the software house developer, but rather by other developers and members of collaborative network who are attracted to work in opensource products and services and will thus help augment the overall resources available to specify and develop the collaborative solutions as opposed to traditional competitors' solutions. These "outside" developers may be motivated by the prospect of working with software tools that solves important problems for them and for others, the possibility of future gain providing related services and creating related products, the opportunity to increase their own personal knowledge, or the ego satisfaction of enhancing their reputation among their peers [21].

Framed in this objective it surfaces the concept of a collaboration portal. This collaboration approach seeks to provide collaboration mechanisms in single place, deploying collaborative capabilities that extend standard business applications.

A leading open-source portal framework for web content management (WCM) is the Portal Liferay®. The Liferay portal is in principle a stack of java portlets that provides a WCM system to support integrity of all functionalities of a collaborative framework. It is a software platform designed for creating dynamic websites and web applications. It can display different page content depending on whether the user is logged in or not. It is a good platform to start with development of collaborative web sites for teams and entire businesses or wiki pages. It also supports Social Networking, Mashups, Content Management and Document sharing out of box with integration with for example Microsoft Office® [22]. 


\section{The Services for Collaborative Networks}

The CoReNet project was conceived in order to address specifically the health sector, considering Textile, Clothing and Footwear (TCFI) consumer's goods for people like elderly, obese, diabetics, and disables. In the case of the TCFI companies, it is necessary to go further and consider cross-sector interactions since some activities like market trends and customers' needs analysis, products design, supplier's management and finite product delivery need to be synchronized and collaboratively integrated. The CoReNet collaborative project responded to such needs by conceiving a new holistic framework, meant as a set of methods, tools and technologies for sustainable small series industrial value creation of health fashionable goods enabling synchronized product design, production and delivery and functional answer to consumer needs. A key element in order to deploy these services for the supply networks stakeholders was the Collaborative Portal. The Collaborative Portal is the virtual place, where actors can find information, support and easy access to the tools and products developed and configured within the collaborative community.

The Collaborative Portal Structure. The Collaborative Portal represents an open space for actors active in the area of target groups like diabetics, obese, elderly and disabled people supporting the usage and validation of the CoReNet results and stimulating the discussions about topics related to personalization, design, production, sustainability of products.

The CoReNet Collaboration Portal is based on social networking approach to provide a friendly and collaborative environment in the Textile, Clothing and Footwear sector: consumers and companies can join different communities testing solutions and sharing needs and interests on healthy and fashionable products for specific market niches. Inside the Portal, the user can find open communities like Textile and Clothing community and Footwear community where it is possible to access demo trials, video and short presentations on innovative tools supporting design, profiling and production of healthy and fashionable products. It is also present four pilot communities for dedicated case studies where specific products are under development according to the needs of the target groups and the specific features of the companies analyzed.

The Fig. 1 presents the schema of the Collaborative Portal structure. This portal is accessed by an Internet connection through a browser application. The application server runs over the web content management framework of Liferay and supports legacy integration and messaging with companies external applications such as ERP's through a Enterprise Service Bus (ESB).

The CoReNet tools represent adaptable and agile instruments specifically tailored for the SMEs in the textile, clothing and footwear sector to support the revision of their business processes in a collaborative way.

CoReNet proposes a novel approach based on matching market needs with fashionable and functional products and related innovative technological solutions for textile, clothing and footwear industries. Within CoReNet Collaborative Portal, all partners of the value chain will be able to actively collaborate in value creation processes where the end consumer is the driving actor. CoReNet methods and tools will enable design changes and production processes adaptation for easy and sustainable product customizations. 


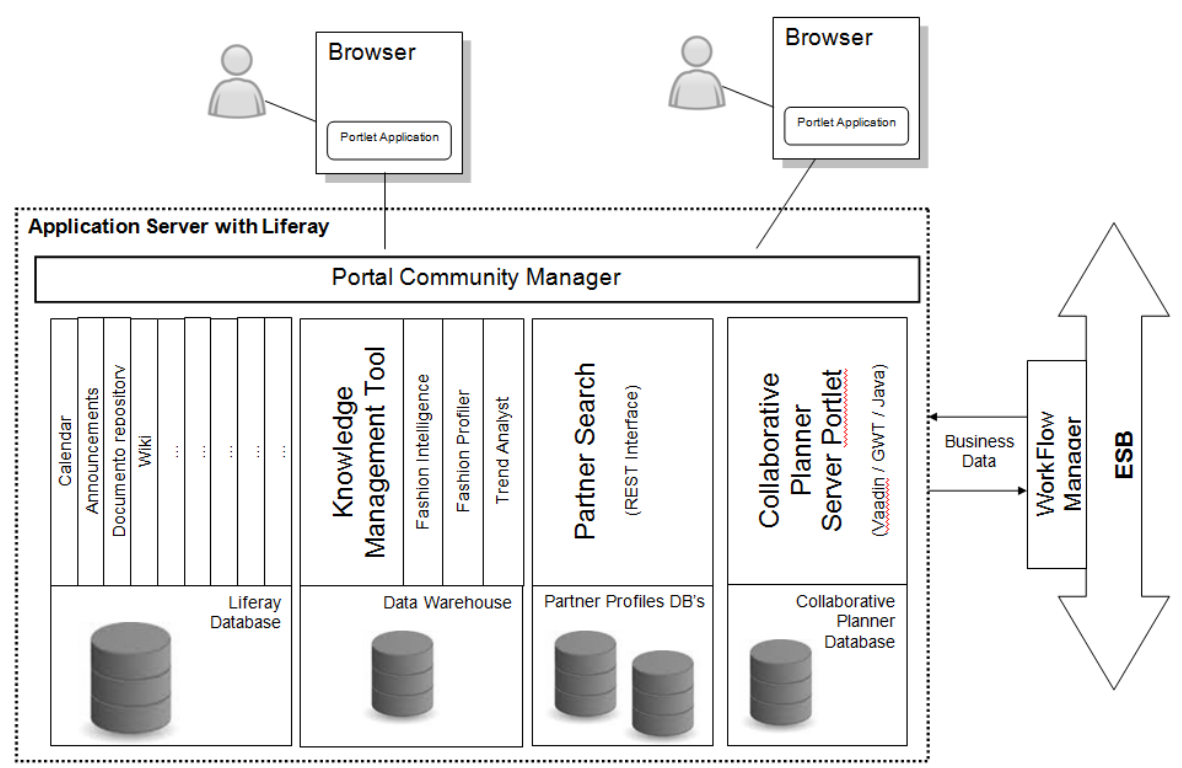

Fig. 1. Collaborative Portal Structure

Management of Market Trend Analysis. The Knowledge Management Tool (KMT) for cross-sectorial trends is a toolset based in new market information model collaboratively sourced from the unstructured information from consumers (aesthetic, physical parameters, comfort and health requirements), retailers (sales status and customer preferences), designers and suppliers interaction (sectorial requests and provisions), and within a social network environment, relying upon the use of semantic technologies for knowledge extraction and pattern matching for consumer trends identification.

The KMT application is composed of several tools and services integrated in the CoReNet innovative consumer-cooperative environment for product design. The components are:

- KMT Fashion Intelligence (FI) - The main features of this tool are: Data mart with transactional data organized for reporting and exploratory data analysis; Data mart with data collected from comments made in social networks (Pinterest.com, in particular) about the products and part of the transactional data. These data are integrated and organized for reporting and exploratory data analysis; Demand characteristics and social network comments exploration using OLAP; Demand characteristics and social network comments reporting using reports and dashboards.

- KMT Fashion Profiler (FP) - The main features of this service are: Automatic segmentation customer demand using clustering algorithms; Visualization of customer demand profiles;

- KMT Trends Analyst (TA) - The main features of this service are: Automatic identification of demand and social network comment trends using association rules mining algorithms; Visualization of demand and social network comment trends; 
Supply Chain Configuration. The Partner Search (PS) is a web-based tool designed to help managers in the supply chain to configure the network by allowing the manufacturers to manage knowledge about its suppliers, by creating and updating Partners Profiles (a set of parameters describing competences and past performance), by visualizing data and key performance indicators (KPIs) and by providing search features to select those partners that best match the entered criteria.

Different typologies of indicators identified are:

- Quantitative indicators are based on historical data relating to the previous and existing relationships of a manufacturer with its partners;

- Qualitative indicators are based on subjective evaluation made by a partner (production managers, purchasing managers, buyers, according to who is in charge of the partner search in the company).

The $P S$ can be used collaboratively after product design, before order collection and at the production planning phase. At these stages it is important to define the configuration of the supply chain for the targeted production season so that the bestsuitable partner is activated on need (i.e any time new orders are collected). In case it is not possible to extrapolate the adequate partner from the list of former ones, the system allows also an open advanced search on the Internet. The Partner Search might be used also after order collection in case there are specific needs of customization and personalization of the product.

Data can be both manually entered by human users, (through a GUI implemented as a portlet) and automatically retrieved from legacy systems (e.g. manufacturer's ERP systems).

Collaborative Planning. The CoReNet Collaborative Planning approach is oriented to address the demand planning and demand fulfillment activities. The tool allows each partner to directly propose new delivery dates, lead times and costs, via a web-based planning graphical tool which is available and shared by all supply network partners.

The planning scheme is based on negotiations undertaken between all the core partners and potential partners. The main goal of this negotiation is to arrive to an optimized plan for the supply network, where all the required operations are characterized in terms of minimum and maximum lead times, minimum and maximum time overlaps and minimum cost, and other relevant negotiation indicators defined at the beginning of the negotiation.

The proposed approach provides several key benefits for manufacturers and suppliers of the TCFI sector that are looking for agile solutions for the order management and the production plan processes supporting the production of small series. The Collaborative Planner solution is:

- easily accessible and easy to use, as the tools provide advanced GUI and are available within a unique portal (thus no installation is required);

- supports the exchange and the automatic check of business information through well-known channels, like the emails (hiding technical details about the internal format of the exchanged documents);

- helps the selection of partners leveraging on information already owned by the manufacturer and provides an open collaborative environment where planning with the selected ones an agreed production plan. 
The proposed planning approach integrates the partner profiling and search services and fits the needs of customer-oriented supply networks in achieving flexibility and responsiveness to the market demands.

\section{Conclusions and Further Research}

The ongoing European project "Customer-oriented and eco-friendly networks for healthy fashionable goods (CoReNet)" aims to provide TCF Industry companies with the tools and methods to face the challenge of working in demand-driven and customer oriented collaborative networks.

During the project test and validation of specific isolated tools on the pilot cases, arose the need to offer a virtual place, where all the actors involved in the collaborative network could find information, interact, obtain support and easy access to the tools and products developed and configured within the collaborative community. This need has led to the design and development of the Collaborative Portal. This new tool, evolved in order to support the network formation, the knowledge sharing and the expertise in manufacturing of small batches of products. The ultimate objective of this collaborative portal became the offer of an integrated set of collaborative services that equip fashionable and healthy footwear \& garments network managers and stakeholders with the necessary guidance to address the combination of processes, functions, activities, relationships and pathways along which products, services and information move in and between TCF companies networks. Until now the project research work allowed the definition of a Collaborative Portal that set up the foundations for the development of future technologies and tools that support network operation and the instantiation of collaborative services. The undergoing phases include the evaluation of the current services and tools and the improvement of the concept inside the CoReNet project together with the industry partners and the customers. The final goal is to provide a sound customer-oriented Collaborative Portal suitable to collaborative network managers, stakeholders of TCF industry and customers.

Acknowledgments. The research leading to these results has received funding from the European Union's Seventh Framework Programme (FP7/2007-2013) under grant agreement $n^{\circ}[260169]$.

\section{References}

1. Camarinha-Matos, L.M., Boucher, X., Afsarmanesh, H. (eds.): PRO-VE 2010. IFIP AICT, vol. 336. Springer, Heidelberg (2010)

2. Christopher, M.: Logistics and supply chain management - Creating Value-Adding Networks, 3rd edn. Prentice Hall (2005)

3. Simchi-Levi, D.: Operations Rules - Delivering Customer Value through Flexible Operations. The MIT Press (2010)

4. Ramirez, R.: Value co-production: intellectual origins and implications for practice and research. Strategic Management Journal 20(1), 49-65 (1999) 
5. Romero, D., Molina, A.: Collaborative networked organisations and customer communities: value co-creation and co-innovation in the networking era. Production Planning \& Control 22(5-6), 447-472 (2011)

6. Kim, S.T.: Implementation of green supply chain management: Impact on performance outcomes in small- and medium-sized electrical and electronic firms. In: Lee, S.M. (ed.) Management, United States - Nebraska (2010)

7. Camarinha-Matos, L.M., Afsarmanesh, H.: Collaborative networks: A new scientific discipline. Journal of Intelligent Manufacturing 16(4-5), 439-452 (2005)

8. Afsarmanesh, H., Camarinha-Matos, L.M.: A framework for management of virtual organization breeding environments. In: Camarinha-Matos, L.M., Afsarmanesh, H., Ortiz, A. (eds.) Collaborative Networks and their Breeding Environments. IFIP, vol. 186, pp. 35-48. Springer, Heidelberg (2005)

9. Kankaanpää, T., et al.: Methodology for non-hierarchical collaboration networks for complex products manufacturing. In: Collaborative Networks for a Sustainable World, vol. 336, pp. 204-211 (2010)

10. Carneiro, L.M., Almeida, R., Azevedo, A.L., Kankaanpaa, T., Shamsuzzoha, A.H.M.: An innovative framework supporting SME networks for complex product manufacturing. In: Camarinha-Matos, L.M., Boucher, X., Afsarmanesh, H. (eds.) PRO-VE 2010. IFIP AICT, vol. 336, pp. 204-211. Springer, Heidelberg (2010)

11. Moore, J.F.: The death of competition: leadership and strategy in the age of business ecosystems. HarperBusiness New York (1996)

12. Chituc, C.-M., Azevedo, A.L.: Multi-perspective challenges on collaborative networks business environments. In: Camarinha-Matos, L.M., Afsarmanesh, H., Ortiz, A. (eds.) Collaborative Networks and their Breeding Environments. IFIP, vol. 186, pp. 25-32. Springer, Heidelberg (2005)

13. Frayret, J.-M.: A network approach to operate agile manufacturing systems. International Journal of Production Economics 74(1-3) (2001)

14. De Maggio, M., Gloor, P.A., Passiante, G.: Collaborative innovation networks, virtual communities and geographical clustering. International Journal of Innovation and Regional Development 1(4), 387-404 (2009)

15. Camarinha-Matos, L.M., Afsarmanesh, H., Ollus, M.: Methods and Tools for Collaborative Networked Organizations. Springer (2008)

16. Romero, D., Molina, A.: Virtual organisation breeding environments toolkit: reference model, management framework and instantiation methodology. Production Planning \& Control 21(2), 181-217 (2010)

17. Lefebvre, É., et al.: E-collaboration within one supply chain and its impact on firms' innovativeness and performance. Information Systems and e-Business Management 1(2), $157-173$ (2003)

18. Walters, D., Rainbird, M.: Cooperative innovation: A value chain approach. Journal of Enterprise Information Management 20(5), 595-607 (2007)

19. Ignatiadis, I., et al.: Introducing a Collaborative Business Model for European ERP Value Chains of SMEs. In: Camarinha-Matos, L., Afsarmanesh, H., Novais, P. (eds.) Establishing the Foundation of Collaborative Networks; IFIP, vol. 243, pp. 505-512. Springer, Boston (2010)

20. Papazoglou, M.P., Van Den Heuvel, W.-J.: Service oriented architectures: approaches, technologies and research issues. The VLDB Journal 16(3), 389-415 (2007)

21. Hecker, F.: Setting up shop: The business of Open-Source software. IEEE Software 16(1), 45-51 (1999)

22. Liferay. Liferay - Enterprise, Open Source, For Life (2014), http: / /www. Iiferay.com/home (cited April 25, 2013) 